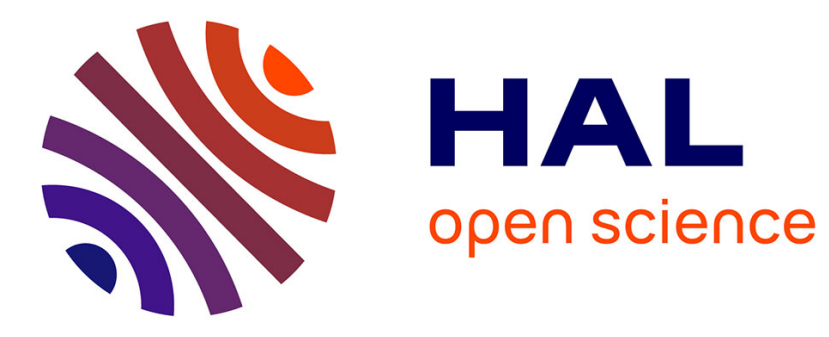

\title{
Sub-wavelength silicon grating metamaterial ring resonators
}

\author{
Daniel Benedikovic, Mathias Berciano, Guillaume Marcaud, Xavier Le Roux, \\ Vladyslav Vakarin, Carlos Alonso-Ramos, Eric Cassan, Delphine \\ Marris-Morini, Laurent Vivien
}

\section{- To cite this version:}

Daniel Benedikovic, Mathias Berciano, Guillaume Marcaud, Xavier Le Roux, Vladyslav Vakarin, et al.. Sub-wavelength silicon grating metamaterial ring resonators. 2018. hal-01876660

\section{HAL Id: hal-01876660 https://hal.science/hal-01876660}

Preprint submitted on 18 Sep 2018

HAL is a multi-disciplinary open access archive for the deposit and dissemination of scientific research documents, whether they are published or not. The documents may come from teaching and research institutions in France or abroad, or from public or private research centers.
L'archive ouverte pluridisciplinaire HAL, est destinée au dépôt et à la diffusion de documents scientifiques de niveau recherche, publiés ou non, émanant des établissements d'enseignement et de recherche français ou étrangers, des laboratoires publics ou privés. 


\title{
Sub-wavelength silicon grating metamaterial ring resonators
}

\author{
Daniel Benedikovic ${ }^{1, *}$, Mathias Berciano ${ }^{1}$, Guillaume Marcaud ${ }^{1}$, Xavier Le Roux ${ }^{1}$, Vladyslav Vakarin ${ }^{1}$, Carlos \\ Alonso-Ramos ${ }^{1}$, Eric Cassan ${ }^{1}$, Delphine Marris-Morini ${ }^{1}$, and Laurent Vivien ${ }^{1}$ \\ ${ }^{1}$ Centre for Nanoscience and Nanotechnology, CNRS, Univ. Paris-Sud, Université Paris-Saclay, C2N - Orsay, 91405 Orsay \\ Cedex, France \\ *daniel.benedikovic@u-psud.fr
}

\begin{abstract}
We report on experimental results of silicon micro-ring resonators based on non-resonant photonic metamaterial waveguides. High extinction ratio up to $30 \mathrm{~dB}$ and loaded Q-factors in a range of 1500 to 6000 were achieved at a wavelength of $1550 \mathrm{~nm}$.
\end{abstract}

Keywords- Ring resonators, silicon photonics, silicon-oninsulator, sub-wavelength grating metamaterials, nanophotonics

\section{INTRODUCTION}

Micro-ring resonators implemented on the well-established silicon-on-insulator (SOI) technology are recognized to be fundamental building blocks for multitude of complex photonic components [1-8]. This includes both passive and active devices such as filters [3], modulators [4], detectors [5], or sensors [6,7]. Most of ring resonators realized on conventional strip-like SOI waveguides can afford high quality factors (Q-factor) and low-loss propagation due to the high modal confinement, enabled by large index contrast of SOI platform. Conversely, this tight optical mode confinement imposes fundamental limitations to explore light-matter interactions with the surrounding environment (claddings).

In order to complementary combine a good mode confinement as well as a good mode interaction with the surrounding medium sub-wavelength grating (SWG) nanostructuration of regular $\mathrm{Si}$ waveguides can be developed [3,6-12]. SWGs, non-resonant nanophotonic structures of dimensions at a scale below the wavelength of light, allow local control of a variety of propagation properties. As demonstrated, this strategy opened up a promising way towards advanced $\mathrm{Si}$ photonic devices, typically achieving performances that substantially outperform their conventional counterparts [9-12]. More recently, only few research works have investigated the potential of SWG metamaterial waveguides to build microring resonators [3,6-8], demonstrating promising prospects for enhanced on-chip functionalities.

Here, we report on experimental demonstration of SOI micro-ring resonators with SWG nanostructuration. The fabricated devices showed a high extinction ratio up to $30 \mathrm{~dB}$ and loaded Q-factors up to $\sim 6000$.

\section{DESIGN AND FABRICATION}

The ring resonator based on SWG metamaterials is schematically shown in Fig. 1a. Devices are implemented on SOI platform, with 220-nm-thick $\mathrm{Si}$ and 2 - $\mu \mathrm{m}$-thick buried oxide (BOX) layers. The ring resonator geometry comprises nominal waveguide width $W=500 \mathrm{~nm}$. The SWG structure is formed by interlacing Si blocks of length $L_{\mathrm{b}}$ and fully etched trenches of length $L_{\mathrm{t}}$. The SWG pitch $\Lambda$ $=L_{\mathrm{b}}+L_{\mathrm{t}}$ is sufficiently small, thereby frustrating the diffraction. The SWG duty cycle is defined as $D C=L_{\mathrm{b}} / \Lambda$. The ring radius is denoted $R$ and the coupling gap is $G_{\mathrm{c}}$. For SWG pitch of $200 \mathrm{~nm}$, and wavelength around $1.55-\mu \mathrm{m}$, the structure operates at deep SWG regime and mimic optically equivalent strip waveguide of reduced refractive index. By alternating the geometry of SWG metamaterial waveguides, optical properties of this non-resonant photonic waveguides such as refractive index, modal confinement, mode profile as well as dispersion can be judiciously controlled.

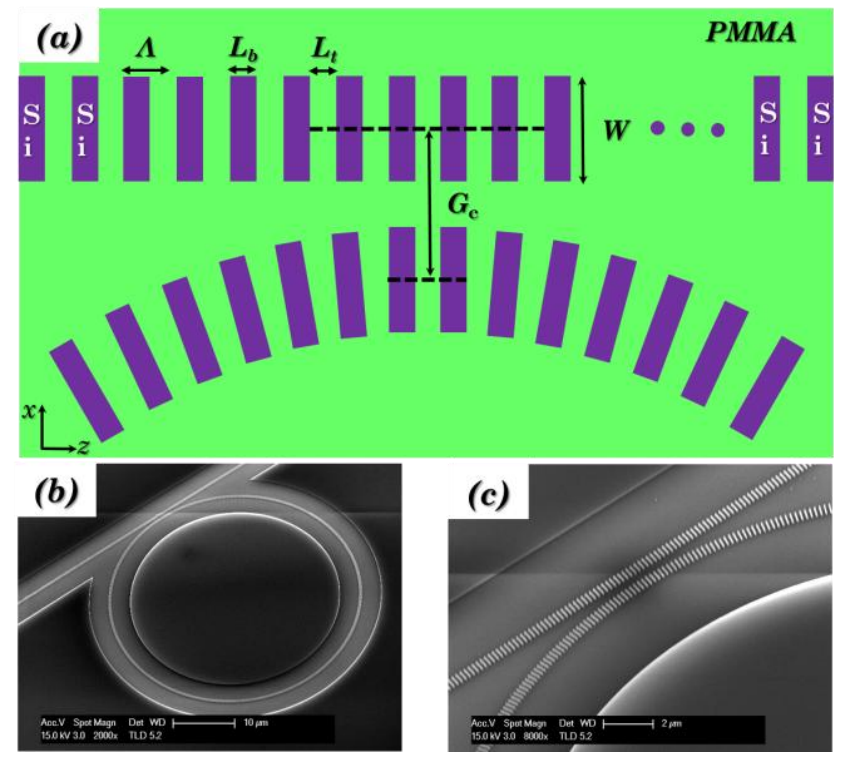

Fig. 1 (a) In-plane schematic view of the SWG-based micro-ring resonator and the bus waveguide. (b) and (c) Scanning electron microscopy images of fabricated device prior to the deposition of upper cladding (PMMA).

We estimated both effective and group indices of such SWG metamaterial waveguides used in ring resonators. Simulations were carried out using two-dimensional (2-D) mode solver, utilizing Finite Element Method (FEM). Figure $2 \mathrm{a}$ shows the evolution of the calculated free spectral range (FSR) as a function of wavelength for different ring radii $(R=10-; 20-;$ and $30-\mu \mathrm{m})$ and its comparison with experimentally retrieved values. From Fig. $2 \mathrm{a}$, it can be observed that the performed calculations and experiments 
are in excellent agreement. This clearly confirms the expected trends of ring resonators based on non-resonant metamaterial waveguides.

Ring resonators were fabricated on standard SOI substrates, with 220-nm Si and 2- $\mu \mathrm{m} \mathrm{BOX} \mathrm{layers,} \mathrm{using} \mathrm{single-etch}$ step process. The devices were first patterned by using electron beam lithography, with a $5 \mathrm{~nm}$ writing pitch. The pattern was then transferred onto the top $\mathrm{Si}$ layer using reactive ion etching. The PMMA was deposited on top of fabricated structures using spin coating to achieve a $3-\mu \mathrm{m}-$ thick overlayer. The scanning electron microscopy (SEM) images of fabricated SWG-based ring resonators before the deposition of the upper cladding are shown in Fig. 1b and c, respectively.
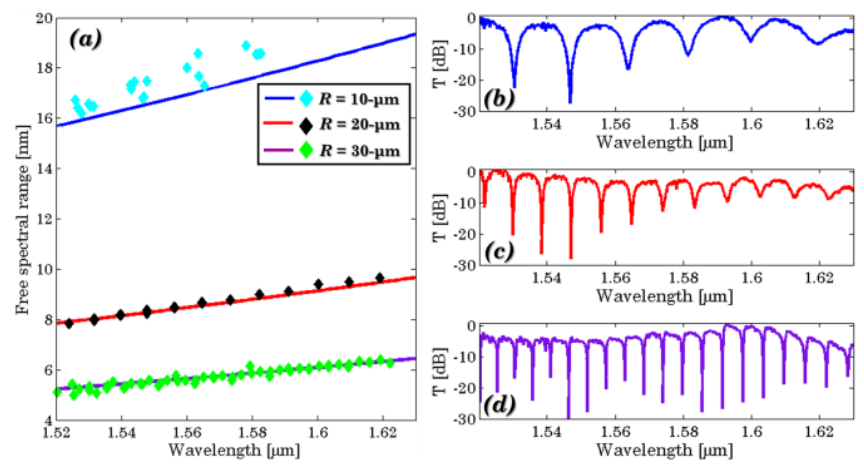

Fig. 2 (a) Simulated (lines) and measured (markers) free spectral range as a function of wavelength for SWG ring resonators of different radiuses. Normalized transmission spectral response of a SWG ring resonator, with (b) $R=10-\mu \mathrm{m}$, (c) $R=20-\mu \mathrm{m}$, and (d) $R=30-\mu \mathrm{m}$.

\section{OPTICAL PERFORMANCE}

In order to facilitate optical testing, we performed back-toback transmission characterization. The light coming from the tunable laser source (1520-1630 nm, swept with a resolution of $10 \mathrm{pm}$ ) was coupled via single-mode optical fiber to and from the Si chip using SWG-engineered surface grating couplers. These were optimized for transverse electrical (TE) polarization and operation near the wavelength of $1.55 \mu \mathrm{m}$ [12]. Mode size transformers were employed to provide smooth transition between the conventional $\mathrm{Si}$ strip waveguide and the patterned bus metamaterial waveguide [9]. The pitch and duty cycle of the converter were $200 \mathrm{~nm}$ and $50 \%$, respectively. The width of the central strip was varied from $500 \mathrm{~nm}$ down to $150 \mathrm{~nm}$, along the $45-\mu \mathrm{m}$-long tapering section.

Figs. 2b-d show measured transmission spectra for different ring resonators of different radii based on SWG metamaterial waveguides. Here, the transmission spectral response was normalized to the response of SWGengineered surface grating couplers. It can be seen that high extinction ratio was achieved, up to $\sim 28 \mathrm{~dB}$. In order to determine the full width at half maximum, and subsequently, to estimate the Q-factor from the measured SWG ring resonators, the transmission spectral responses were fitted to a Lorentzian function using a least-square fitting approach. Different way of estimating FWHMs and Q- factors was reported in Ref. [3,8], yielding a comparatively different value of those parameters. Figure $3 a-c$ shows the normalized transmission spectral responses nearby the respective resonance wavelengths, for different SWG-based ring resonators, with ring radii of $10-; 20-$; and $30-\mu \mathrm{m}$. The estimated loaded Q-factor is in range of $\sim 1700$ to $\sim 6000$.

Since, the SWG nanostructuration of Si waveguides affords additional design freedom to control all relevant propagation properties such as mode profile and confinement as well as refractive index and overall waveguide dispersion, we are working on improving both the ring resonator designs as well as fabrication fidelity to explore the true potential of SWG-based resonant components.

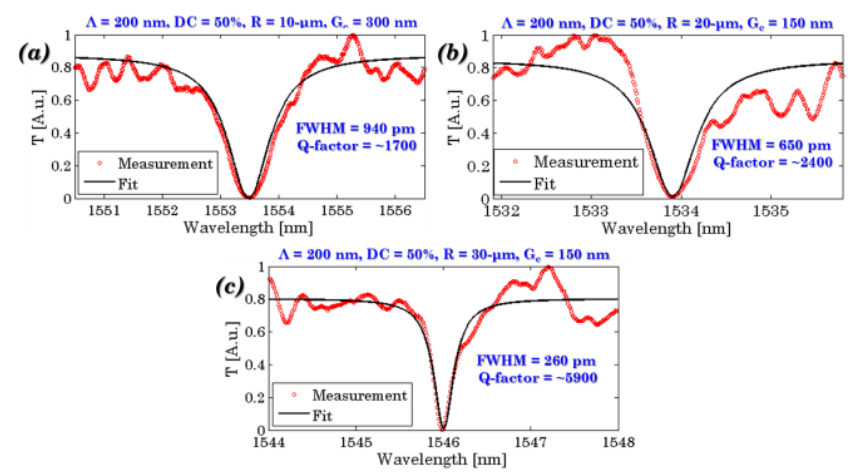

Fig. 3 Normalized transmission spectrum to the respective resonances of various SWG-based ring resonators. Resonance wavelength: (a) $\lambda_{0}=$ $1553.500 \mathrm{~nm}$, (b) $\lambda_{0}=1533.900 \mathrm{~nm}$, and (c) $\lambda_{0}=1546.020 \mathrm{~nm}$.

\section{CONCLUSION}

In this work, we experimentally demonstrated the SOI micro-ring resonators based on non-resonant SWG metamaterial waveguides. The fabricated devices shown a high extinction ratio as high as $-30 \mathrm{~dB}$ and loaded Q-factors up to $\sim 6000$.

\section{ACKNOWLEDGMENTS}

This work was supported by the European Research Council (ERC) under the European Union's Horizon 2020 research and innovation program (ERC POPSTAR - grant agreement No 647342).

\section{REFERENCES}

[1] W. Bogaerts, et al., Laser Photon Rev 6(1), 47-73 (2012).

[2] L. W. Luo, et al., Opt. Express 19(7), 6284-6289 (2011).

[3] J. Wang, et al., Opt. Express 22(13), 15335-15345 (2014).

[4] G. T. Reed, et al., Nat. Photonics 4(8), 518-526 (2010).

[5] D. F. Logan, et al., IEEE Photon. Technol. Lett. 22(20), 1530-1532 (2010).

[6] V. Donzela, et al., Opt. Express 23(4), 4791-4803 (2015).

[7] J. Flueckiger, et al., Opt. Express 24(14), 15672-15686 (2016).

[8] Z. Wang, et al., Opt. Lett. 41(14), 3375-3378 (2016).

[9] P. Cheben, et al., Opt. Lett. 35(15), 2526-2528 (2010).

[10] R. Halir, et al., Laser Photon Rev 9(1), 25-49 (2015).

[11] J. G. Wanguemert-Perez, et al., Opt. Lett. 39(15), 4442-4445 (2014).

[12] D. Benedikovic, et al., Laser Photon Rev 8(6), 93-94 (2014). 